\title{
Nanocrystalline Silicon and Silicon Carbide Optical Properties
}

\author{
Daria Lizunkova ${ }^{1}$, Natalya Latukhina ${ }^{1}$, Victor Chepurnov ${ }^{1}$ and Vyacheslav Paranin ${ }^{1}$ \\ ${ }^{1}$ Samara National Research University, 34, Moskovskoe shosse, Samara, 443086, Russia
}

\begin{abstract}
Porous silicon posseses a wide range of the unique properties and has good perspectives for photo-sensitive structures for a solar cells new generation. Due to the developed pore system, the area of absorbing surface increases, and also the increased sensitivity expands into the short-wavelength region due to the increased energy band gap of silicon nano-particles and silicon nano-filaments on the walls of the pores. Carbonization of the surface layer of porous silicon makes absorption even more effective. In this case, the spectrum of the solar cell expands into the shortwavelength region due to absorption of the high-energy photons in the wide gap material ( $\mathrm{SiC}$ ). In this study, layers of nanocrystalline porous silicon and porous silicon carbide are used as wide-gap material layers in photosensitive structures. The spectral characteristics of the specular reflectance of these materials are investigated.
\end{abstract}

Keywords: porous silicon, photoluminescence, rare earth elements,, photoelectric converters, silicon carbide, reflection coefficient

\section{Introduction}

The use of porous silicon (por-Si) as a sensitive layer in multilayer heterostructures of silicon photoelectric converters makes it possible to significantly increase the efficiency of energy conversion [1]. A promising sensitive layer of a photoelectric converters is a layer with silicon nanocrystals, as well as layers of wide-band materials. At the same time, the absorption spectrum of the photoconductivity spectrum expands into the short-wavelength region due to the quantum-size increase in the width of the band gab of silicon in nanocrystals and due to the absorption of high-energy photons in the wide-band material. An effective system of silicon nanocrystals can be a layer of porous silicon, since the pore walls are a disordered system of quantum wells, filaments and quantum dots [2]. In addition, due to the developed pore system, the area of the absorbing surface of the photodetector increases significantly. However, a number of existing problems prevents the use of porous silicon in the photoelectric converters. This low reproducibility of results due to uncontrolled factors of the technological process, instability of the PC parameters due to the reagent remaining in its pores, as well as its high electrical resistance. The solution to these problems can be the creation of a porous layer locally on the surface with seeds of pore formation, as well as the use of a stabilizing coating, which can be a wide-band silicon carbide semiconductor. The aim of this work was to study the photoelectric properties of samples of multilayer photosensitive structures with a porous layer locally created on the working surface and a stabilizing coating of silicon carbide. The porous layer was created on silicon substrates with textured and ground surfaces. The seeds of pore formation on the ground and textured surfaces are the depressions of the microrelief, where the electric-field intensity is maximum, so a porous layer on such surfaces is formed locally [3]. Samples with a polished surface have served as tested ones. Some samples were carbidized, that is, an epitaxial layer of silicon carbide was created on the surface of the porous layer, so that the samples were $\mathrm{Si} / \mathrm{SiC}$ heterostructures with a large area of the absorbing surface.

\section{Experimental technique}

To create a porous layer, silicon plates were subjected to electrochemical etching in a vertical cell in water-alcohol solutions of hydrofluoric acid. 
Carbidization of the samples leading to the formation of SiC / Si heterostructures was carried out by gas-transfer endotaxy in a hydrogen stream in a vertical reactor with cold walls using a graphite container [4].

The measurements included the measurement of the specular reflection coefficient and the structures photoluminescence. The photoluminescence spectra were measured by excitation with an ultraviolet ( $330 \mathrm{~nm}$ ) laser at the room temperature for samples with a porous layer doped with rare-earth elements (REE) such as erbium or ytterbium.

The spectral dependences of the reflection coefficients were studied using a Shimadzu UV-2450 spectrophotometer with a prefix 206-14046. The measurement range was $0.3-1 \mu \mathrm{m}$, the measurement step and the spectral width of the monochromator slit were $2 \mathrm{~nm}$, the scanning rate was slow. The angle of radiationincidencehaving an elliptical polarization of about 3: $1-4: 1$ was $5^{\circ}$ with an aperture of not more than $5^{\circ}$. The radiation receiver of the Shimadzu UV-2450 spectrophotometer is a photoelectric multiplier. This causes significant noise measurements of the instrument in the near infrared region.

\section{Morphology}

Figure 1 shows SEM images of transverse cleavages of samples with a porous layer formed on thepolished (a) and textured (b) surfaces. On the SEM image of the surface of the textured layer in the region of the junction of the pyramids, it is clearly seen that the porous layer was formed predominantly in the depression of the relief (the darker areas in Fig. 1, c). The sample with a textured surface has undergone carbidisation, as a result of which nanowires of carbon clearly visible on the cleaved surface were formed in some regions of the surface. The porous layer thickness of this sample was $12.55 \mu \mathrm{m}$.

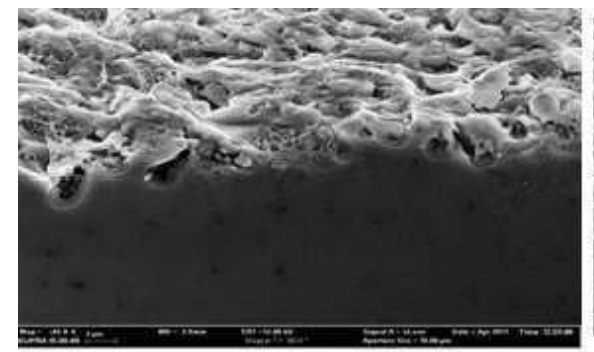

a)

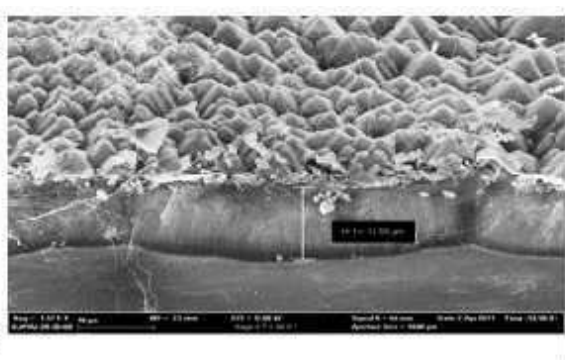

b)

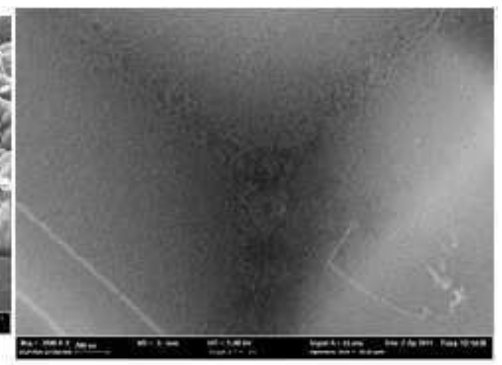

c)

Figure 1: SEM images of transverse cleavages of samples with a porous layer formed on (a) the porous layer is formed on groundsurfaces; (b) the porous layer is formed ontextured surfaces; (c) the image of a textured surface in the area on the pyramidsjoint, where a porous layer was formed.

\section{Spectral dependences of the reflection coefficient}

The spectral dependences of the reflection coefficients were studied using a SHIMADZU UV-2450PC spectrophotometer in the wavelength range from 0.3 to $1 \mu \mathrm{m}$ on different types of the working surface of the samples. Samples with a porous surface (past electrochemical etching), as well as samples that have undergone carbidization, have entered the measuring group. KDB-3 silicon plates with textured, ground or polished surfaces without pores were used as test samples. We can see that the formation of a porous layer significantly reduces the reflection coefficient, while the course of the curves of the spectral dependences remains almost unchanged, which is explained by the local nature of pore formation. An exception is a sample with a textured surface that was etched for 5 minutes, its reflectivity coefficient in the short-wave part of the spectrum is noticeably lower than in others ones. This is explained by pore formation dynamics on such surface. At the initial stage of etching, silicon nanocrystals are formed almost over the entire surface of the textured layer, and with further etching some of them located on the walls of the pyramids dissolve, and the formation of the porous layer only occurs in the relief depressions [5]. A similar course of the curve for 
the spectral dependence of the reflection coefficient is also observed for carbided samples with a "failure" of the reflection coefficient in the 250 - $300 \mathrm{~nm}$ x-band. It is explained by the absorption of light in nanocrystals of wide-band silicon carbide [6].

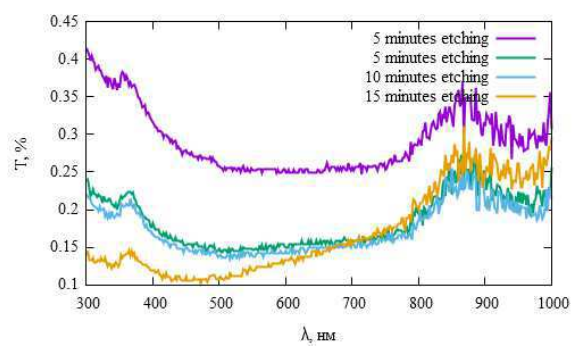

a)

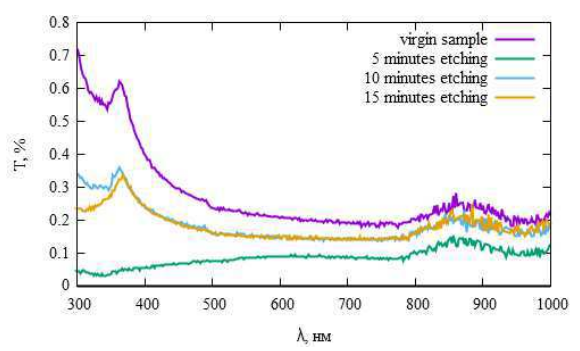

b)

Figure 2: Spectral dependences of the reflection coefficients of samples with a porous layer formed at different etching time on: (a) ground surface; (b)textured surface.

\section{Spectral dependences of photoluminescence}

Figure 3 shows the photoluminescence spectra of porous silicon samples doped with ytterbium (a) and erbium (b), where narrow peaks of the spectral maxima of ytterbium emission at $980 \mathrm{~nm}$ and erbium emission at $1550 \mathrm{~nm}$ are clearly visible. Since the mechanism of photoluminescence of REE ions in a solid silicon matrix is based on the recombination of an exciton generated by radiation in a silicon nanocrystal, the presence of sufficiently intense peaks of PL of ytterbium and erbium in the spectra of the samples under study confirms the presence of a sufficiently large concentration of nanocrystals in porous layers [7]. In figure 3, a wide band of $550750 \mathrm{~nm}$ corresponding to the emission spectrum of silicon nanocrystals is also visible, as well as a laser pumping peak at $370 \mathrm{~nm}$.

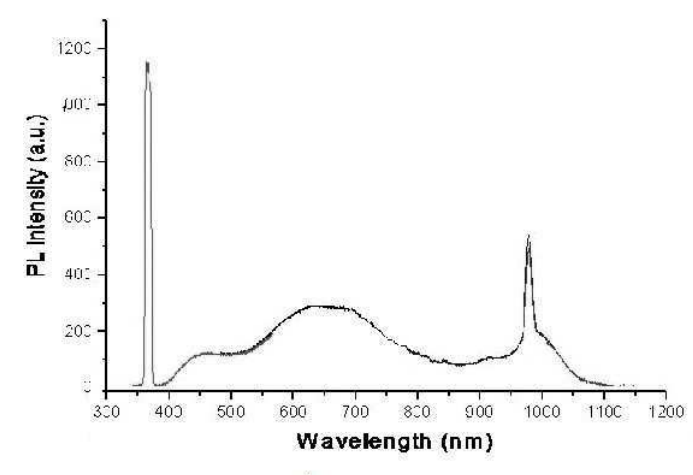

a)

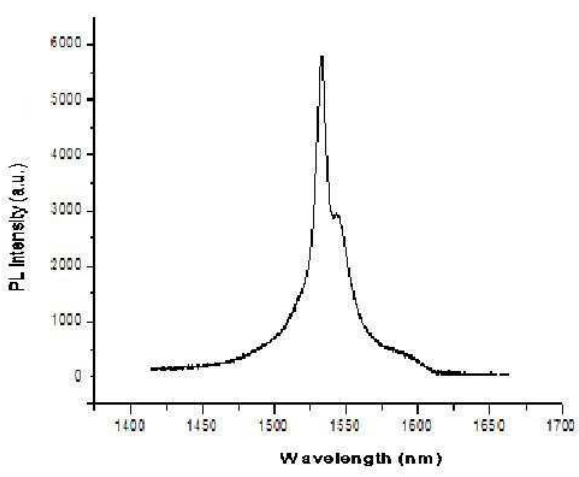

b)

Figure 3: Photoluminescence spectra of samples with a layer of porous silicon formed on a textured surface: a) doped with ytterbium; b) doped with erbium.

\section{A reflecting surface model}

The working surface of the photosensitive structures can be represented as a consisting of two components one with different reflection coefficients: textured and porous. The microrelief on the textured surface of silicon is an etching polyhedron in the form of regular tetragonal pyramids with lateral faces that are natural surfaces of a single crystal and an angle at the apex of $70.5^{\circ}$. The textured surface reduces optical losses due to the total effect of multiple reflection of the incident beam from the frontal surface and multiple total internal reflection from the back and side 
surfaces. The trajectories of light rays on an idealized textured surface with the refractive index of the medium $\mathrm{n}=1$ are shown in Figure 4. The light normally incident to the surface undergoes several reflections, as a result of which the intensity of the reflected light will decrease as a power of multiplicity. The nature of the interaction of the incident radiation with such a surface will strongly depend on the relationship between the wavelength and the geometric dimensions of the relief. While the geometric dimension of the microrelief exceeds the wavelength of the radiation, the laws of geometric optics operate, that is, multiple effects reflection take place here. If the height of the relief is comparable with the wavelength or much less than the latter, then with respect to this radiation the surface is perfectly smooth and manifests itself as highly reflective. When reflecting from the microrelief surfaces in the area where the radiation "feels" the microrelief along with the mirror component of the reflected light, there is also a diffusely scattered constituent. Measurements made in the wavelength range 0.5-1.2 $\mu$ m give the value $\mathrm{R}=5-7 \%$. The application of multilayer antireflection coatings makes it possible to reduce the reflection coefficient almost to zero [8].

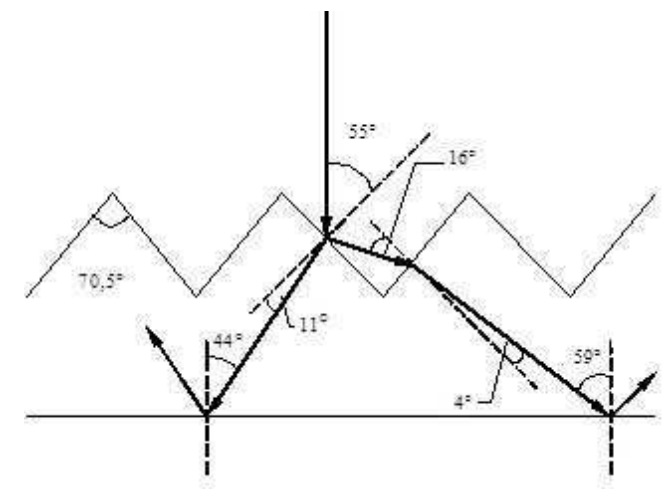

Figure 4: A trajectory of thelight rays on the idealized textured surface of the solar cell with refractive indices of the medium $\mathrm{n}=1$ and $\mathrm{n}_{S i}=3.8$.

According to the data of [9], the reflection coefficient can be determined from the formula:

$$
R=(1-\delta) r_{1} r_{2}+\delta r_{1} r_{2} r_{3}
$$

Where is a part of the secondary reflected light, $r_{1}, r_{2}, r_{3}$ are reflection coefficients from the successive faces, depending on the light wavelength. Since the faces of the pyramids are atomically smooth surfaces oriented along the crystallographic plane (111), the numerical values of these coefficients can take the values of the reflection coefficients from the polished silicon surface for a given wavelength. The reflectivity of the surface of porous silicon depends strongly on its porosity. With a large degree of porosity, the reflection coefficient of the porous layer can be practically zero throughout the visible range of wavelengths of the incident radiation.

Let is consider, for convenience of calculation, an ideal textured surface, averaging the heights of the pyramids:

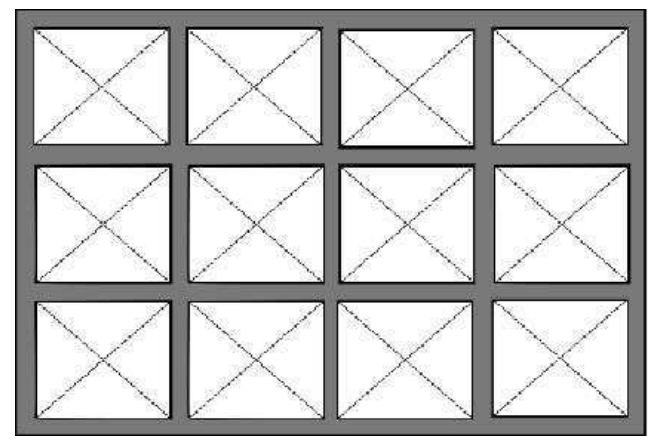

Figure 5: A schematic representation of the ideal surface (top view) 
The height of these pyramids was $2.26 \mu \mathrm{m}$, base $-3.23 \mu \mathrm{m}$; the width of the regions of the porous layer (gray in Figure 5) is $1.25 \mu \mathrm{m}$. Calculations of the reflection coefficient were carried out for a surface, $56.97 \%$ of the area occupied by a textured surface and $43.03 \%$ of the surface area of a porous layer (Fig. 6).

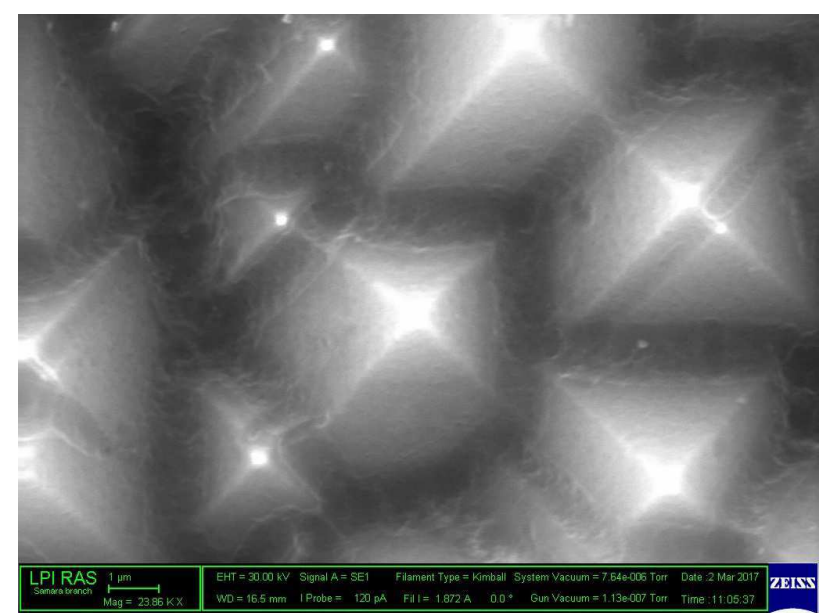

a)

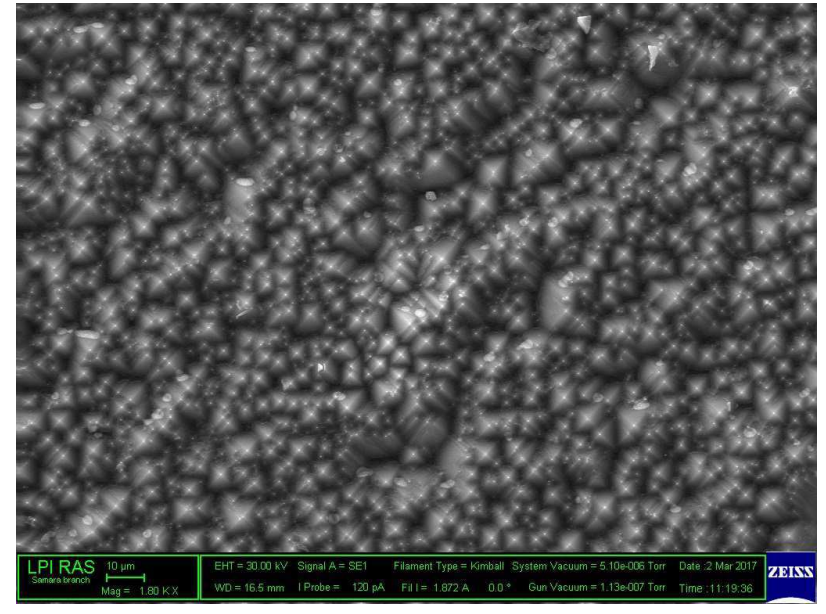

b)

Figure 6: SEM images of the simulated surface on a different scale

The numerical values of the area shares were determined using the program JMicroVision v1.27 (Fig. 7).

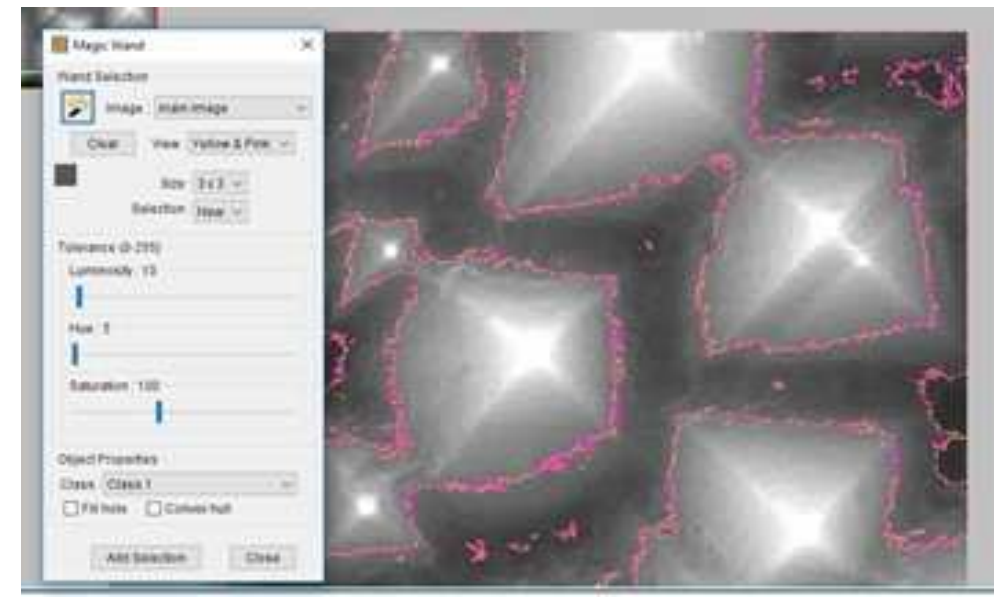

Figure 7: Isolation of regions with different reflectivity on the investigated surface

The simulation results are shown in Fig. 8. The reflection coefficient $\mathrm{R}$ is calculated at an angle of incidence of light to the first face $=54^{\circ} 40^{\prime}$, to the opposite face $=16^{\circ}$, a small part of the secondary reflected light $(=10 \%)$ returns to the first face at an angle of $86^{\circ} 40^{\prime}$. The calculation is made for wavelengths of $0.4-0.75 \mu \mathrm{m}$. When taking into account the illumination of different faces, the reflection coefficient from the textured part of the surface is calculated by the formula:

$$
R=0.35\left[r_{1} r_{2}(1-\delta)+\delta r_{1} r_{2} r_{3}\right]+0.65 r_{1}^{\prime} r_{2}^{\prime},
$$

Indices and strokes for $r$ correspond to the notation for the angles of incidence. The coefficient of reflection from the porous part of the surface was assumed to be zero throughout the investigated wavelength range.

Analyzing the obtained data, we can say that the reflection coefficient, experimentally determined, at a given degree of filling is much lower than the theoretical one. This can be explained by the fact that the real faces of the 


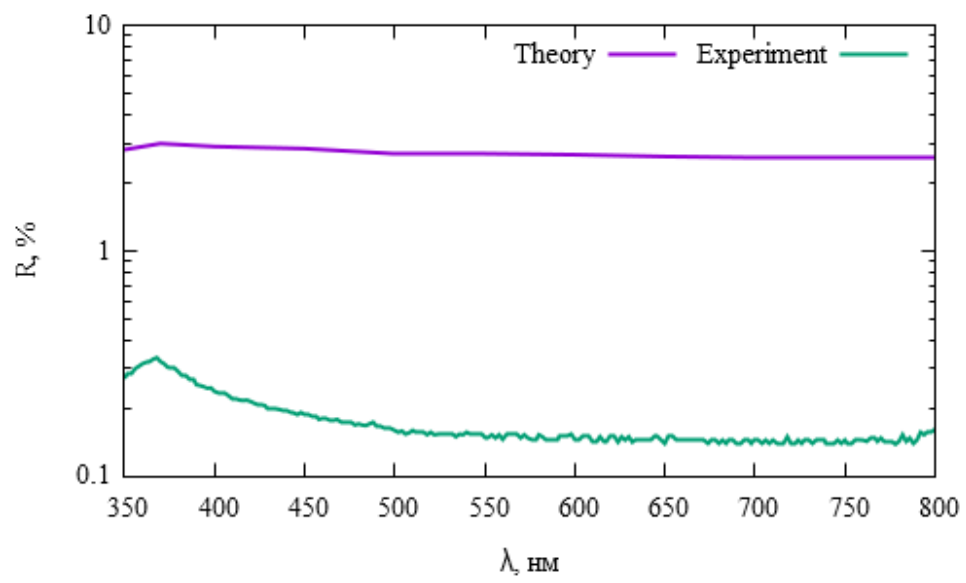

Figure 8: Spectral dependence of the reflection coefficient of samples with a fillability of $43 \%$ of the surface by a porous layer, both theoretical and experimental curves

pyramids are not atomically smooth. As a result of electrochemical etching, a weakly expressed relief appears on them. It significantly reduces their reflection coefficient, which is also observed in the works of other authors [10]. It should be noted that the values of the experimental reflection coefficient for a textured silicon surface with a porous layer, obtained in [10], are much higher than those ones obtained in this work. It may be explained by a significantly smaller fraction of the surface occupied by the porous layer.

\section{Conclusion}

Thus, the conducted studies show that porous silicon, created on a textured or polished surface, is an effective system of nanocrystals. Its application in a photoconductive device makes it possible to increase significantly the fraction of absorbed radiation in the spectral range 400-1000 $\mathrm{nm}$ and to increase the photocurrent.

\section{References}

[1] Latukhina, N. Efficient silicon solar cells for space and ground-based aircraft./ N. Latukhina, A. Rogozin, G. Puzyrnaya, D. Lizunkova, A. Gurtovb, S. Ivkov // ProcediaEngineering. 2015.- Vol. 104. -p. 157-161

[2] Latukhina, N.V. New prospects of old materials: silicon and silicon carbide / N.V. Latukhina, V.I. Chepurnov, G.A. Pisarenko // Electronics of the NTB. - 2013. - 4 (00126) - p.104-110.

[3] Sokolov, V.I. Some characteristics of porous silicon (reflection, scattering, refractive index, microhardness) / V.I. Sokolov, A.I. Shelnyh. // JETP letters. -2008-V.34, . 5. - p. 34-39.

[4] Latukhina, N.V. Photosensitive Heterostructures on the Basis of Nanocrystal Porous Silicon/N. V. Latukhina, A. S. Rogozhin, S. Saed, V. I. Chepurnov// Russian Microelectronics, 2016, Vol. 45, Nos. 89, pp. 613618

[5] Gosteva E.A. Investigation of the coefficient in silicon structures with different porosity ./ E.A. Gosteva, V.V. Starkov, Yu. N. Parkhomenko // Nanostructured materials and conversion devices for solar energy: a collection of proceedings of the IV All-Russian Scientific Conference (September 29-30, 2016, Cheboksary), 2016, p.59-63

[6] Kirsanov, N. Yu. Multilayer Photosensitive Structures Based on Porous Silicon and Rare-Earth-Element Compounds: Study of Spectral Characteristics/ N. Yu. Kirsanov , N. V. Latukhina, D. A. Lizunkova, G. A. Rogozhina, and M. V. Stepikhova// Semiconductors, 2017, Vol. 51, No. 3, pp. 353356

[7] Sokolov S.A. Photoluminescence of Rare Earth Ions (Er3+, Yb3+) in a Porous Silicon Matrix/ S. A. Sokolov, R. Rsslhuber, D.M. Zhigunov, N.V. Latukhina, V.Yu. Timoshenko // Thin Solid Films, 2014, V.562, p. $462-466$

[8] Gorbach T.Ya.Selective properties of an anisotropically etched surface/ T.Ya. Gorbach, S.V. Svechnikov, N.V. Kotova, E.V. Podlisny // Optoelectronics and Semiconductor Technology, 1986. V.10. - p. 649.

[9] Borodina N.M. Silicon photoconverters with a textured surface and their properties / N.M. Borodina, A.K. Zaitseva, E.A. Marasanova, A. A. Polisman // Helio Technique, 1982. 3, p.6-11.

[10] Hyukyong Kwon. Investigation of Antireflective Porous Silicon Coating forSolar Cells/ Hyukyong Kwon, Jaedoo Lee, Minjeong Kim, and Soohong Lee// International Scholarly Research Network ISRN Nanotechnology, V. 2011, p.1-4 


\title{
Nanocrystalline Silicon and Silicon Carbide Optical Properties
}

\author{
Daria Lizunkova ${ }^{1}$, Natalya Latukhina ${ }^{1}$, Victor Chepurnov ${ }^{1}$ and Vyacheslav Paranin ${ }^{1}$ \\ ${ }^{1}$ Samara National Research University, 34, Moskovskoe shosse, Samara, 443086, Russia
}

\begin{abstract}
Porous silicon posseses a wide range of the unique properties and has good perspectives for photo-sensitive structures for a solar cells new generation. Due to the developed pore system, the area of absorbing surface increases, and also the increased sensitivity expands into the short-wavelength region due to the increased energy band gap of silicon nano-particles and silicon nano-filaments on the walls of the pores. Carbonization of the surface layer of porous silicon makes absorption even more effective. In this case, the spectrum of the solar cell expands into the shortwavelength region due to absorption of the high-energy photons in the wide gap material ( $\mathrm{SiC}$ ). In this study, layers of nanocrystalline porous silicon and porous silicon carbide are used as wide-gap material layers in photosensitive structures. The spectral characteristics of the specular reflectance of these materials are investigated.
\end{abstract}

Keywords: porous silicon, photoluminescence, rare earth elements,, photoelectric converters, silicon carbide, reflection coefficient

\section{Introduction}

The use of porous silicon (por-Si) as a sensitive layer in multilayer heterostructures of silicon photoelectric converters makes it possible to significantly increase the efficiency of energy conversion [1]. A promising sensitive layer of a photoelectric converters is a layer with silicon nanocrystals, as well as layers of wide-band materials. At the same time, the absorption spectrum of the photoconductivity spectrum expands into the short-wavelength region due to the quantum-size increase in the width of the band gab of silicon in nanocrystals and due to the absorption of high-energy photons in the wide-band material. An effective system of silicon nanocrystals can be a layer of porous silicon, since the pore walls are a disordered system of quantum wells, filaments and quantum dots [2]. In addition, due to the developed pore system, the area of the absorbing surface of the photodetector increases significantly. However, a number of existing problems prevents the use of porous silicon in the photoelectric converters. This low reproducibility of results due to uncontrolled factors of the technological process, instability of the PC parameters due to the reagent remaining in its pores, as well as its high electrical resistance. The solution to these problems can be the creation of a porous layer locally on the surface with seeds of pore formation, as well as the use of a stabilizing coating, which can be a wide-band silicon carbide semiconductor. The aim of this work was to study the photoelectric properties of samples of multilayer photosensitive structures with a porous layer locally created on the working surface and a stabilizing coating of silicon carbide. The porous layer was created on silicon substrates with textured and ground surfaces. The seeds of pore formation on the ground and textured surfaces are the depressions of the microrelief, where the electric-field intensity is maximum, so a porous layer on such surfaces is formed locally [3]. Samples with a polished surface have served as tested ones. Some samples were carbidized, that is, an epitaxial layer of silicon carbide was created on the surface of the porous layer, so that the samples were $\mathrm{Si}$ / SiC heterostructures with a large area of the absorbing surface.

\section{Experimental technique}

To create a porous layer, silicon plates were subjected to electrochemical etching in a vertical cell in water-alcohol solutions of hydrofluoric acid. 
Carbidization of the samples leading to the formation of SiC / Si heterostructures was carried out by gas-transfer endotaxy in a hydrogen stream in a vertical reactor with cold walls using a graphite container [4].

The measurements included the measurement of the specular reflection coefficient and the structures photoluminescence. The photoluminescence spectra were measured by excitation with an ultraviolet ( $330 \mathrm{~nm}$ ) laser at the room temperature for samples with a porous layer doped with rare-earth elements (REE) such as erbium or ytterbium.

The spectral dependences of the reflection coefficients were studied using a Shimadzu UV-2450 spectrophotometer with a prefix 206-14046. The measurement range was $0.3-1 \mu \mathrm{m}$, the measurement step and the spectral width of the monochromator slit were $2 \mathrm{~nm}$, the scanning rate was slow. The angle of radiationincidencehaving an elliptical polarization of about 3: $1-4: 1$ was $5^{\circ}$ with an aperture of not more than $5^{\circ}$. The radiation receiver of the Shimadzu UV-2450 spectrophotometer is a photoelectric multiplier. This causes significant noise measurements of the instrument in the near infrared region.

\section{Morphology}

Figure 1 shows SEM images of transverse cleavages of samples with a porous layer formed on thepolished (a) and textured (b) surfaces. On the SEM image of the surface of the textured layer in the region of the junction of the pyramids, it is clearly seen that the porous layer was formed predominantly in the depression of the relief (the darker areas in Fig. 1, c). The sample with a textured surface has undergone carbidisation, as a result of which nanowires of carbon clearly visible on the cleaved surface were formed in some regions of the surface. The porous layer thickness of this sample was $12.55 \mu \mathrm{m}$.

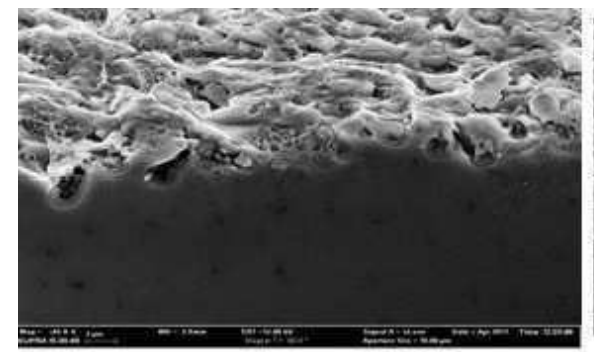

a)

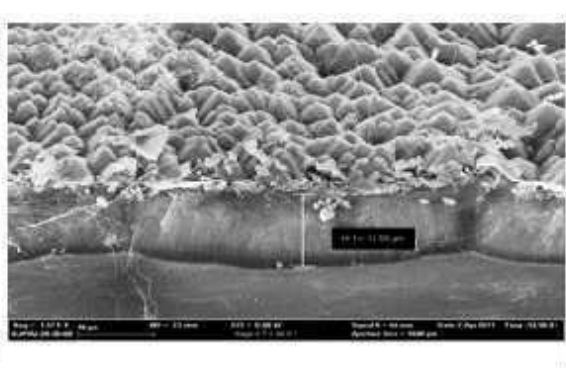

b)

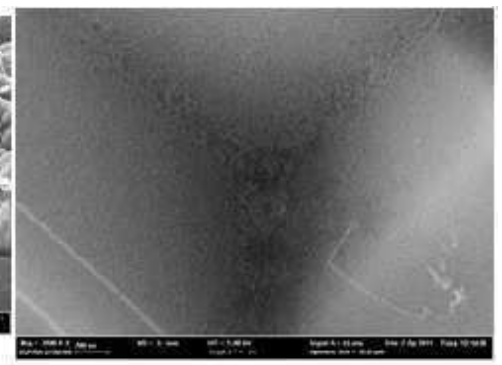

c)

Figure 1: SEM images of transverse cleavages of samples with a porous layer formed on (a) the porous layer is formed on groundsurfaces; (b) the porous layer is formed ontextured surfaces; (c) the image of a textured surface in the area on the pyramidsjoint, where a porous layer was formed.

\section{Spectral dependences of the reflection coefficient}

The spectral dependences of the reflection coefficients were studied using a SHIMADZU UV-2450PC spectrophotometer in the wavelength range from 0.3 to $1 \mu \mathrm{m}$ on different types of the working surface of the samples. Samples with a porous surface (past electrochemical etching), as well as samples that have undergone carbidization, have entered the measuring group. KDB-3 silicon plates with textured, ground or polished surfaces without pores were used as test samples. We can see that the formation of a porous layer significantly reduces the reflection coefficient, while the course of the curves of the spectral dependences remains almost unchanged, which is explained by the local nature of pore formation. An exception is a sample with a textured surface that was etched for 5 minutes, its reflectivity coefficient in the short-wave part of the spectrum is noticeably lower than in others ones. This is explained by pore formation dynamics on such surface. At the initial stage of etching, silicon nanocrystals are formed almost over the entire surface of the textured layer, and with further etching some of them located on the walls of the pyramids dissolve, and the formation of the porous layer only occurs in the relief depressions [5]. A similar course of the curve for 
the spectral dependence of the reflection coefficient is also observed for carbided samples with a "failure" of the reflection coefficient in the 250 - $300 \mathrm{~nm}$ x-band. It is explained by the absorption of light in nanocrystals of wide-band silicon carbide [6].

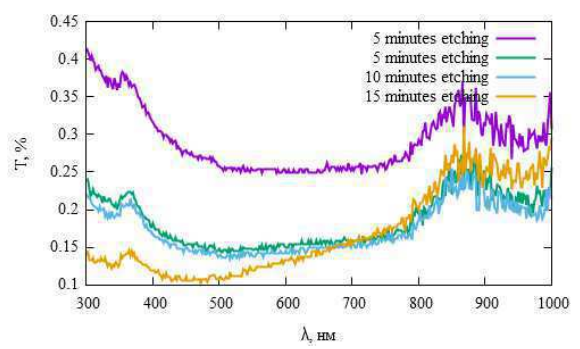

a)

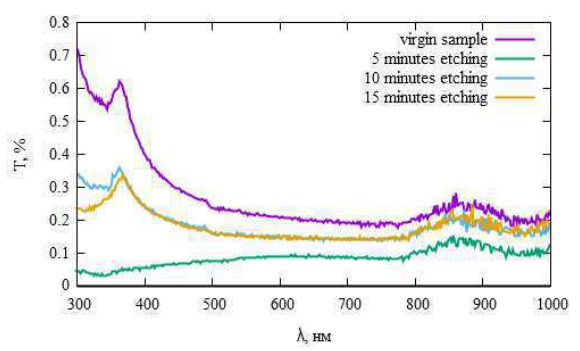

b)

Figure 2: Spectral dependences of the reflection coefficients of samples with a porous layer formed at different etching time on: (a) ground surface; (b)textured surface.

\section{Spectral dependences of photoluminescence}

Figure 3 shows the photoluminescence spectra of porous silicon samples doped with ytterbium (a) and erbium (b), where narrow peaks of the spectral maxima of ytterbium emission at $980 \mathrm{~nm}$ and erbium emission at $1550 \mathrm{~nm}$ are clearly visible. Since the mechanism of photoluminescence of REE ions in a solid silicon matrix is based on the recombination of an exciton generated by radiation in a silicon nanocrystal, the presence of sufficiently intense peaks of PL of ytterbium and erbium in the spectra of the samples under study confirms the presence of a sufficiently large concentration of nanocrystals in porous layers [7]. In figure 3, a wide band of $550750 \mathrm{~nm}$ corresponding to the emission spectrum of silicon nanocrystals is also visible, as well as a laser pumping peak at $370 \mathrm{~nm}$.

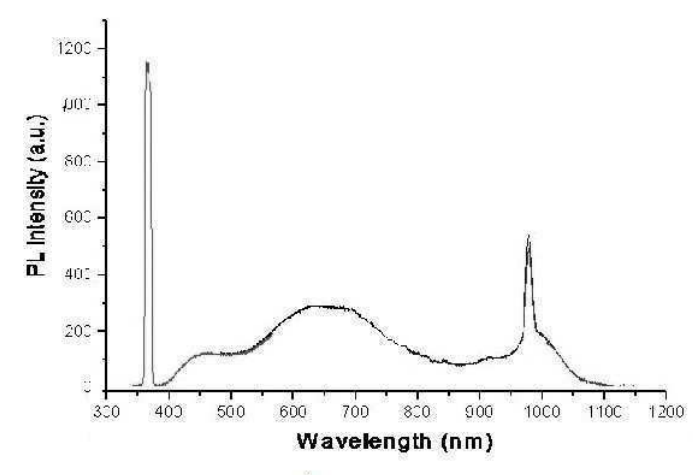

a)

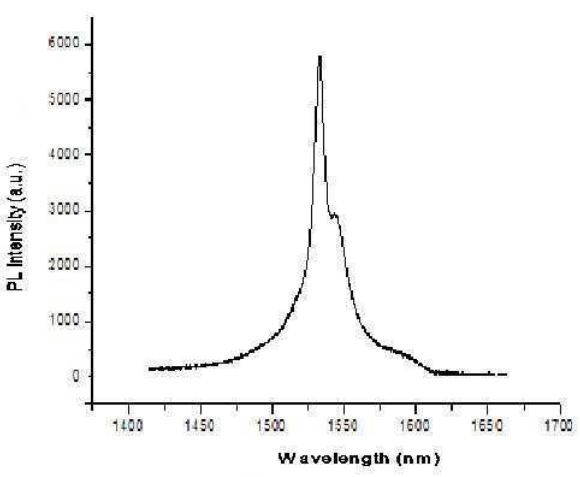

b)

Figure 3: Photoluminescence spectra of samples with a layer of porous silicon formed on a textured surface: a) doped with ytterbium; b) doped with erbium.

\section{A reflecting surface model}

The working surface of the photosensitive structures can be represented as a consisting of two components one with different reflection coefficients: textured and porous. The microrelief on the textured surface of silicon is an etching polyhedron in the form of regular tetragonal pyramids with lateral faces that are natural surfaces of a single crystal and an angle at the apex of $70.5^{\circ}$. The textured surface reduces optical losses due to the total effect of multiple reflection of the incident beam from the frontal surface and multiple total internal reflection from the back and side 
surfaces. The trajectories of light rays on an idealized textured surface with the refractive index of the medium $\mathrm{n}=1$ are shown in Figure 4. The light normally incident to the surface undergoes several reflections, as a result of which the intensity of the reflected light will decrease as a power of multiplicity. The nature of the interaction of the incident radiation with such a surface will strongly depend on the relationship between the wavelength and the geometric dimensions of the relief. While the geometric dimension of the microrelief exceeds the wavelength of the radiation, the laws of geometric optics operate, that is, multiple effects reflection take place here. If the height of the relief is comparable with the wavelength or much less than the latter, then with respect to this radiation the surface is perfectly smooth and manifests itself as highly reflective. When reflecting from the microrelief surfaces in the area where the radiation "feels" the microrelief along with the mirror component of the reflected light, there is also a diffusely scattered constituent. Measurements made in the wavelength range 0.5-1.2 $\mu$ m give the value $\mathrm{R}=5-7 \%$. The application of multilayer antireflection coatings makes it possible to reduce the reflection coefficient almost to zero [8].

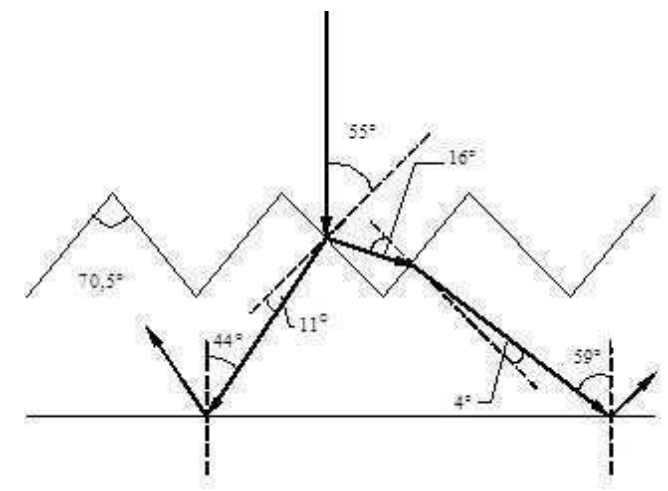

Figure 4: A trajectory of thelight rays on the idealized textured surface of the solar cell with refractive indices of the medium $\mathrm{n}=1$ and $\mathrm{n}_{S i}=3.8$.

According to the data of [9], the reflection coefficient can be determined from the formula:

$$
R=(1-\delta) r_{1} r_{2}+\delta r_{1} r_{2} r_{3}
$$

Where is a part of the secondary reflected light, $r_{1}, r_{2}, r_{3}$ are reflection coefficients from the successive faces, depending on the light wavelength. Since the faces of the pyramids are atomically smooth surfaces oriented along the crystallographic plane (111), the numerical values of these coefficients can take the values of the reflection coefficients from the polished silicon surface for a given wavelength. The reflectivity of the surface of porous silicon depends strongly on its porosity. With a large degree of porosity, the reflection coefficient of the porous layer can be practically zero throughout the visible range of wavelengths of the incident radiation.

Let is consider, for convenience of calculation, an ideal textured surface, averaging the heights of the pyramids:

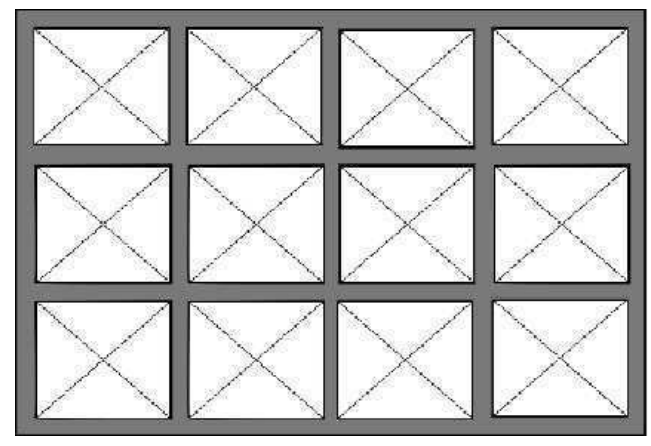

Figure 5: A schematic representation of the ideal surface (top view) 
The height of these pyramids was $2.26 \mu \mathrm{m}$, base $-3.23 \mu \mathrm{m}$; the width of the regions of the porous layer (gray in Figure 5) is $1.25 \mu \mathrm{m}$. Calculations of the reflection coefficient were carried out for a surface, $56.97 \%$ of the area occupied by a textured surface and $43.03 \%$ of the surface area of a porous layer (Fig. 6).

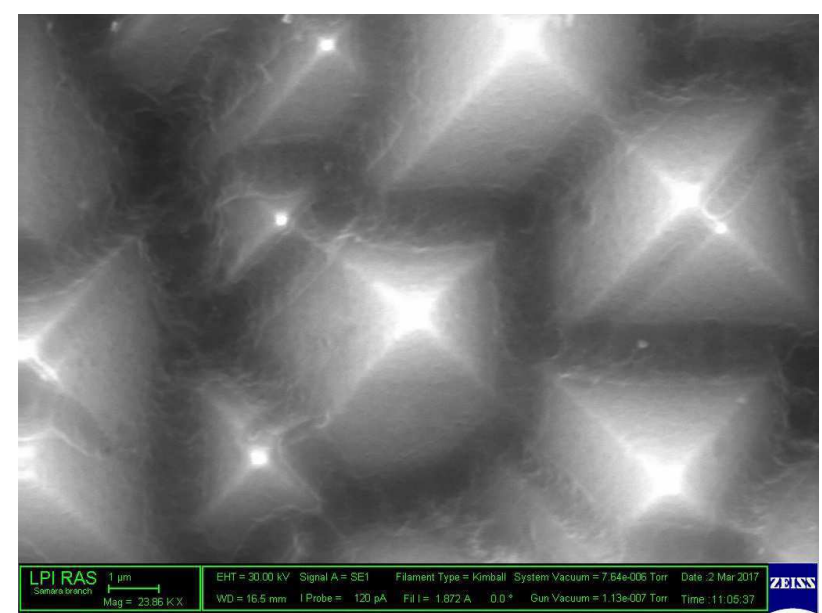

a)

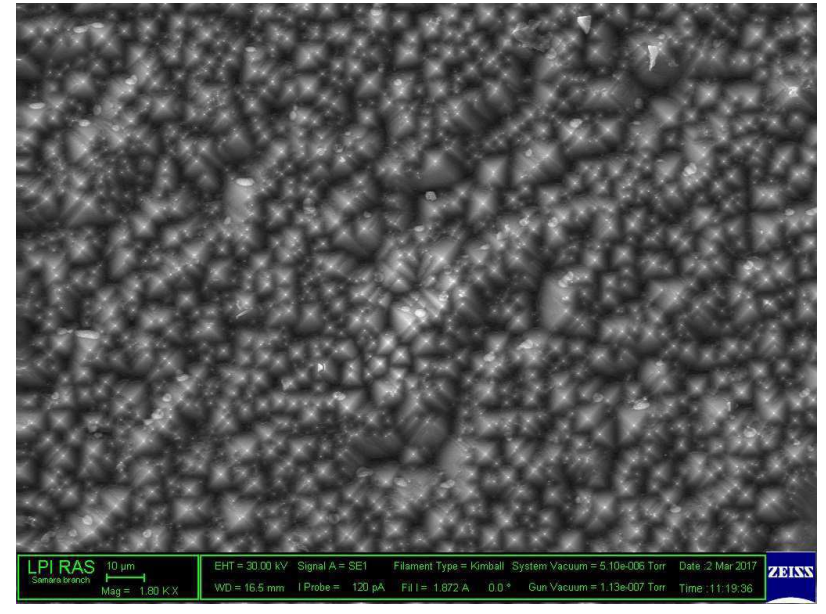

b)

Figure 6: SEM images of the simulated surface on a different scale

The numerical values of the area shares were determined using the program JMicroVision v1.27 (Fig. 7).

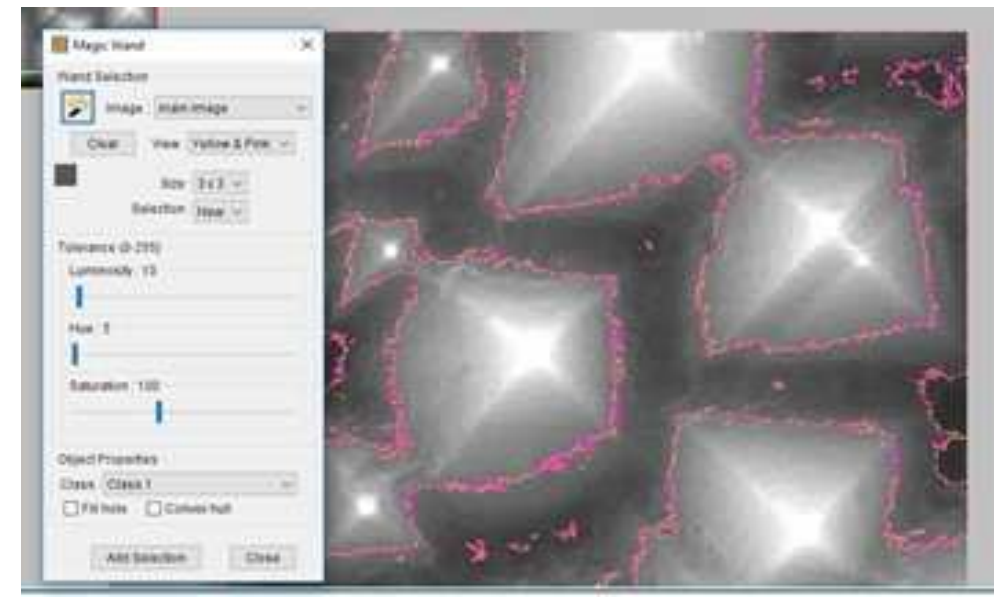

Figure 7: Isolation of regions with different reflectivity on the investigated surface

The simulation results are shown in Fig. 8. The reflection coefficient $\mathrm{R}$ is calculated at an angle of incidence of light to the first face $=54^{\circ} 40^{\prime}$, to the opposite face $=16^{\circ}$, a small part of the secondary reflected light $(=10 \%)$ returns to the first face at an angle of $86^{\circ} 40^{\prime}$. The calculation is made for wavelengths of $0.4-0.75 \mu \mathrm{m}$. When taking into account the illumination of different faces, the reflection coefficient from the textured part of the surface is calculated by the formula:

$$
R=0.35\left[r_{1} r_{2}(1-\delta)+\delta r_{1} r_{2} r_{3}\right]+0.65 r_{1}^{\prime} r_{2}^{\prime},
$$

Indices and strokes for $r$ correspond to the notation for the angles of incidence. The coefficient of reflection from the porous part of the surface was assumed to be zero throughout the investigated wavelength range.

Analyzing the obtained data, we can say that the reflection coefficient, experimentally determined, at a given degree of filling is much lower than the theoretical one. This can be explained by the fact that the real faces of the 


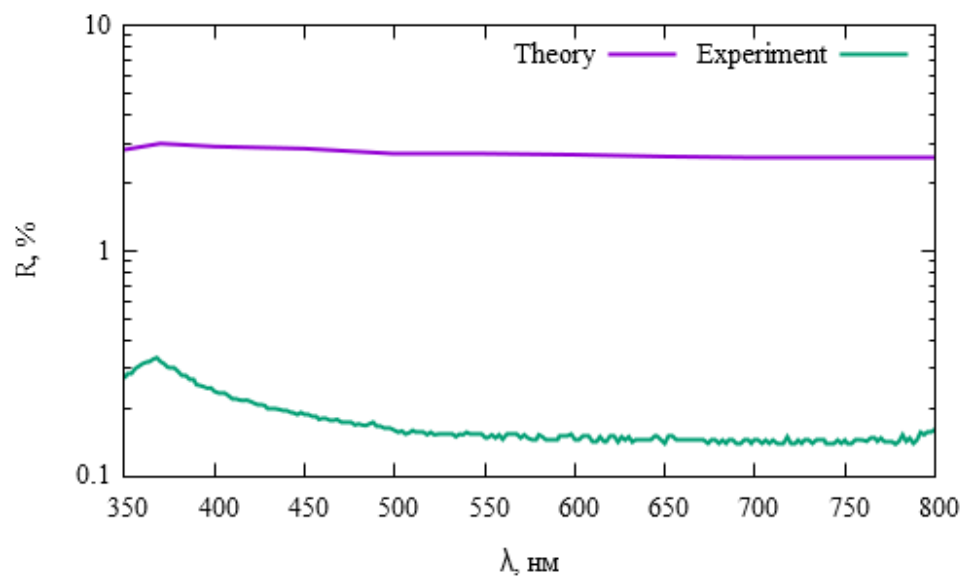

Figure 8: Spectral dependence of the reflection coefficient of samples with a fillability of $43 \%$ of the surface by a porous layer, both theoretical and experimental curves

pyramids are not atomically smooth. As a result of electrochemical etching, a weakly expressed relief appears on them. It significantly reduces their reflection coefficient, which is also observed in the works of other authors [10]. It should be noted that the values of the experimental reflection coefficient for a textured silicon surface with a porous layer, obtained in [10], are much higher than those ones obtained in this work. It may be explained by a significantly smaller fraction of the surface occupied by the porous layer.

\section{Conclusion}

Thus, the conducted studies show that porous silicon, created on a textured or polished surface, is an effective system of nanocrystals. Its application in a photoconductive device makes it possible to increase significantly the fraction of absorbed radiation in the spectral range 400-1000 $\mathrm{nm}$ and to increase the photocurrent.

\section{References}

[1] Latukhina, N. Efficient silicon solar cells for space and ground-based aircraft./ N. Latukhina, A. Rogozin, G. Puzyrnaya, D. Lizunkova, A. Gurtovb, S. Ivkov // ProcediaEngineering. 2015.- Vol. 104. -p. 157-161

[2] Latukhina, N.V. New prospects of old materials: silicon and silicon carbide / N.V. Latukhina, V.I. Chepurnov, G.A. Pisarenko // Electronics of the NTB. - 2013. - 4 (00126) - p.104-110.

[3] Sokolov, V.I. Some characteristics of porous silicon (reflection, scattering, refractive index, microhardness) / V.I. Sokolov, A.I. Shelnyh. // JETP letters. -2008-V.34, . 5. - p. 34-39.

[4] Latukhina, N.V. Photosensitive Heterostructures on the Basis of Nanocrystal Porous Silicon/N. V. Latukhina, A. S. Rogozhin, S. Saed, V. I. Chepurnov// Russian Microelectronics, 2016, Vol. 45, Nos. 89, pp. 613618

[5] Gosteva E.A. Investigation of the coefficient in silicon structures with different porosity ./ E.A. Gosteva, V.V. Starkov, Yu. N. Parkhomenko // Nanostructured materials and conversion devices for solar energy: a collection of proceedings of the IV All-Russian Scientific Conference (September 29-30, 2016, Cheboksary), 2016, p.59-63

[6] Kirsanov, N. Yu. Multilayer Photosensitive Structures Based on Porous Silicon and Rare-Earth-Element Compounds: Study of Spectral Characteristics/ N. Yu. Kirsanov , N. V. Latukhina, D. A. Lizunkova, G. A. Rogozhina, and M. V. Stepikhova// Semiconductors, 2017, Vol. 51, No. 3, pp. 353356

[7] Sokolov S.A. Photoluminescence of Rare Earth Ions (Er3+, Yb3+) in a Porous Silicon Matrix/ S. A. Sokolov, R. Rsslhuber, D.M. Zhigunov, N.V. Latukhina, V.Yu. Timoshenko // Thin Solid Films, 2014, V.562, p. $462-466$

[8] Gorbach T.Ya.Selective properties of an anisotropically etched surface/ T.Ya. Gorbach, S.V. Svechnikov, N.V. Kotova, E.V. Podlisny // Optoelectronics and Semiconductor Technology, 1986. V.10. - p. 649.

[9] Borodina N.M. Silicon photoconverters with a textured surface and their properties / N.M. Borodina, A.K. Zaitseva, E.A. Marasanova, A. A. Polisman // Helio Technique, 1982. 3, p.6-11.

[10] Hyukyong Kwon. Investigation of Antireflective Porous Silicon Coating forSolar Cells/ Hyukyong Kwon, Jaedoo Lee, Minjeong Kim, and Soohong Lee// International Scholarly Research Network ISRN Nanotechnology, V. 2011, p.1-4 